\title{
Avaliação do programa de controle da tuberculose em cenário Amazônico: desafios em Coari
}

\author{
Nicolás Esteban Castro HEUFEMANN¹, Maria Jacirema Ferreira GONÇALVES², Maria Luiza GARNELO³ \\ RESUMO
}

Pesquisa avaliativa, tipo estudo de caso, do contexto e grau de implantaçáo do Programa de Controle da Tuberculose (PCT) no município de Coari, Amazonas, 2001-2008. As técnicas de pesquisa conjugaram abordagens quantitativa e qualitativa, com uso de modelo lógico e matriz de julgamento. O componente quantitativo utilizou dados do Sistema de Informaçáo de Agravos de Notificação e dados populacionais do Departamento de Informática do Sistema Único de Saúde. As variáveis foram, idade, sexo, forma clínica, procedência, tipo de entrada, meios diagnósticos e situação de encerramento. Para a população foram feitos cálculos de interpolaçấo geométrica, por faixa etária, sexo e procedência (urbana e rural). O componente qualitativo combinou técnicas e fontes: pesquisa documental; observação e entrevista. $\mathrm{O}$ modelo lógico propiciou o entendimento da estrutura, processo e resultado do PCT; e a matriz de julgamento permitiu identificar o grau de implantaçáo. A taxa média de incidência da tuberculose na área urbana foi 27 casos por 100.000 habitantes e de 9,4 por 100.000 habitantes na área rural, com razão masculino/feminino de 1,6. A avaliação do contexto externo, vigilância epidemiológica e operacionalização do PCT mostrou grau de implantaçáo intermediário. No contexto organizacional, estrutura física das Unidades Saúde, recursos humanos e efetividade, o resultado foi incipiente. No geral, o grau de implantação foi intermediário. A flutuação nas notificaçóes e taxas anuais de incidência, associada ao náo cumprimento das metas do PCT, à desarticulação entre o PCT e o restante da atenção básica e à não interiorização da assistência indicam sérias dificuldades na operacionalização das ações de controle da tuberculose.

PalavRaS-CHAVE: Atenção Primária à Saúde; Tuberculose; Avaliação de Programas e Projetos de saúde

\section{Evaluation of a tuberculosis control program in an Amazonian scenario: challenges in Coari}

\begin{abstract}
This is an evaluative research, a type of study case of the context and degree of implementation of the Tuberculosis Control Program (PCT) in the city of Coari, State of Amazonas, 2001-2008. Research techniques combined quantitative and qualitative approaches, using a logical model and a judgment matrix. The quantitative component used data from the Information System for Notifiable Diseases and Population Data from the Department of the Brazilian National Health System. The variables assessed were age, sex, clinical form, origin area, type of entry, diagnostic and outcome. For population there were calculated geometric interpolation, by age, gender and residence (urban or rural). The qualitative component combined techniques and sources: documentary research and observation and interview. The logical model led to the understanding of the structure, process and outcome of the PCT, and with the judgment matrix we identified the degree of implementation. The mean tuberculosis incidence was 27 cases per 100.000 inhabitants in urban area, and 9.4 per 100,000 in rural area, with a male / female ratio of 1.6. The external context assessment, surveillance and operation of the PCT showed intermediate degree of implementation. In the organizational context, the physical structure of the Health Units, human resources and effectiveness, the result was incipient. In general, the level of implementation was intermediate. Oscillations in notifications and annual rates of incidence indicate serious difficulties in the actions of the tuberculosis control. These difficulties are also associated with non-compliance with the goals of the PCT, disconnection between the PCT and the primary health care and absence of health care polices for the interior population.
\end{abstract}

KEYWORDS: Primary Health Care; Tuberculosis; Program Evaluation

\footnotetext{
1 Universidade do Estado do Amazonas. Programa de Pós-Graduação Multiinstitucional Mestrado em Saúde, Sociedade e Endemias na Amazônia da Universidade Federal do Amazonas, Universidade Federal do Pará e Instituto de Pesquisas Leônidas \& Maria Deane - FIOCRUZ. Rua Teresina, 476. Adrianópolis, Manaus - Amazonas. CEP 69057-070. E-mail: niescah@ig.com.br

2 Escola de Enfermagem de Manaus/ Universidade Federal do Amazonas (UFAM). Programa de Pós-Graduação Multiinstitucional Mestrado em Saúde, Sociedade e Endemias na Amazônia da Universidade Federal do Amazonas, Universidade Federal do Pará e Instituto de Pesquisas Leônidas \& Maria Deane - FIOCRUZ. Rua Teresina, 495. Adrianópolis, Manaus - Amazonas. CEP 69057-070. E-mail: jaciremagoncalves@ufam.edu.br

${ }^{3}$ Centro de Pesquisas Leônidas \& Maria Deane, Fundação Oswaldo Cruz, Manaus, Brasil. Programa de Pós-Graduação Multiinstitucional Mestrado em Saúde, Sociedade e Endemias na Amazônia da Universidade Federal do Amazonas, Universidade Federal do Pará e Instituto de Pesquisas Leônidas \& Maria Deane - FIOCRUZ. Rua Teresina, 476. Adrianópolis, Manaus - Amazonas. CEP 69057-070. E-mail: luiza.garnelo@amazonia.fiocruz.br
} 


\section{INTRODUÇÃO}

A tuberculose (TB) permanece como um dos principais problemas de saúde a ser enfrentado no mundo. Contribuem para este fato as desigualdades sociais e econômicas, a falta de novos tratamentos e vacinas e as deficiências dos sistemas de saúde (Barreira e Grangeiro 2007), particularmente no que diz respeito aos limites na qualidade e efetividade das açóes de controle deste agravo. O funcionamento adequado dos serviços e programas de saúde, com avaliaçóes periódicas, é requisito fundamental para o controle deste agravo, mas persistem lacunas na avaliaçáo e monitoramento das açôes de controle da TB (Vieira-da-Silva et al. 2007).

O Brasil adotou o Programa Nacional de Controle da Tuberculose (PNCT) como estratégia de controle desta endemia. Ele tem como metas reduzir o abandono do tratamento a menos que $5 \%$, detectar $70 \%$ dos casos pulmonares bacilíferos e curar $85 \%$ dos casos notificados. $\mathrm{O}$ alcance dessas metas, de acordo com o Ministério da Saúde (Jamal e Moherdaui 2007; Brasil 2010a), depende da incorporação do PNCT na rede de atenção básica à saúde, razáo pela qual a avaliaçáa do processo de implantaçáo e operacionalização do programa pode contribuir para aprimorar o controle da endemia tuberculosa.

A avaliação de programas de saúde deve ser entendida como ferramenta cotidiana de gestão e de qualificação dos programas e serviços (Tanaka e Melo 2004; Bezerra et al. 2009). Neste estudo se utiliza a definição de avaliação como um julgamento ex-post de intervenção, usando métodos científicos para empreender a análise de implantação do programa, visando apoiar a tomada de decisão (Contandriopoulos et al. 1997; Tanaka e Melo 2000). Segundo Contandriopoulos et al. (1997), este modelo possibilita a superaçáo do modelo funcionalista tipo "caixa-preta", criticado pela incapacidade de captar a influência do contexto adjacente e os processos de apropriação e adaptação das políticas ou programas pelos agentes que as operacionalizam (Denis e Champagne 1997).

Estudo descritivo do cenário epidemiológico nacional, apoiado nos dados e indicadores do PNCT, mostra que eles não são adequados à realidade das localidades de pequeno porte, nem dos municípios priorizados pelo programa e assinala o potencial da Atenção Primária para a abordagem global do problema da TB (Gonçalves e Penna 2007). Estudos na Amazônia legal encontraram elevados coeficientes de incidência da doença, além de graves problemas na operacionalização do PNCT, comprometendo a efetividade do programa e inviabilizando a redução do agravo (Natal et al. 2004; Moreira et al. 2006; Souza e Pinheiro 2009).

Embora relevantes tais informaçóes são insuficientes para identificar contextos locais de alta vulnerabilidade, demandando novas pesquisas sobre a aplicação do Programa de Controle da TB em âmbito municipal (PCT) e sobre realidades específicas em que a TB represente um grave problema de saúde. Este é o caso de Coari, um importante polo de desenvolvimento econômico microrregional no Amazonas, localizado a $363 \mathrm{~km}$ de Manaus, capital do estado. O PIB de Coari era de $\mathrm{R} \$ 895,92$ milhóes, o segundo maior do estado do Amazonas em 2008 (Amazonas, 2008), contrastando com um Índice de Desenvolvimento Humano (IDH) de médio desenvolvimento humano (de 0,627), o trigésimo terceiro do estado, com renda média mensal de $\mathrm{R} \$ 164,50$ e per capita de R\$ 110,97 (Mourão, Rivas \& Fraxe, 2007). A dinâmica populacional se caracterizou por um crescimento demográfico acelerado, com taxa média de crescimento anual de 6,31\% entre 1991 e 2000, passando de 38.678 em 1991 para 67.096 habitantes em 2000, seguida de um decréscimo populacional de -0,40 entre 2000 e 2007 (Cruz e Teixeira, 2010), com um total de 65.222 habitantes em 2007, segundo a contagem do IBGE (2007). A economia do município tem sido impulsionada pela exploração de petróleo e gás natural da Província Petrolífera de Urucu, em contexto de elevado impacto ambiental e problemas sanitários de grande magnitude, principalmente na malha urbana do município (Almeida e Souza 2008).

A rede de saúde de Coari está habilitada pela Norma Operacional Básica 96 em Gestão Plena do Sistema Municipal (GPSM), devendo se responsabilizar pela oferta de açôes de saúde na Atenção Básica à Saúde (ABS) e na Média Complexidade. De acordo com dados do Cadastro Nacional de Estabelecimentos de Saúde (CNES), a rede básica de serviços dispóe de 11 Unidades Básicas de Saúde (UBS), todas contando com Equipes de Saúde da Família (ESF), que proveem uma cobertura aproximada de $50 \%$ da população do município (BRASIL, 2010b); em 10 dessas unidades o PCT é considerado implantado desde 2001.

O contexto descrito, que congrega acelerada dinâmica demográfica, grande geração de riqueza em pólo de desenvolvimento econômico regional ao lado de significativas desigualdades sociais, é favorável à emergência da TB como importante problema sanitário a ser investigado. Paralelamente, a escassez da literatura voltada para avaliação de açóes de controle de $\mathrm{TB}$ em municípios amazônicos também reforça a justificativa para a realização deste estudo, que avaliou o contexto e grau de implantação do PCT no município de Coari, Amazonas, entre 2001 e 2008.

\section{MATERIAL E MÉTODOS}

Pesquisa avaliativa e exploratória, tipo estudo de caso único, conforme Denis e Champagne (1997), desenvolvida mediante o uso de modelo lógico, com associação de abordagens quantitativas e qualitativas. $\mathrm{O}$ componente quantitativo teve como fontes de dados as notificaçóes de 
casos de TB da base estadual do Sistema de Informaçáo de Agravos de Notificação (SINAN) para o município de Coari-AM. Foram incluídos os casos novos de TB, de ambos os sexos e todas as idades, notificados no período de 2001 a 2008, excluídas as duplicidades, transferências e mudanças de diagnóstico. As variáveis foram idade, sexo, forma clínica, procedência, tipo de entrada, meios diagnósticos e situação de encerramento. As transferências foram excluídas porque não permitiam a avaliaçấo de todas as variáveis propostas para a avaliação.

O componente qualitativo compreendeu uma combinação de técnicas e fontes, tal como orientado por Turato (2003) congregando pesquisa documental dos relatórios anuais de gestão da Secretaria Municipal de Saúde (SEMSA-Coari) de 2007 e 2008, os únicos disponíveis no período estudado; observaçấo das rotinas de atendimento do PCT nas UBS que fazem atendimento de TB e coleta de entrevistas junto aos profissionais de saúde que desenvolvem açóes no PCT.

A seleção das unidades pesquisadas obedeceu à situação local. Das 11 UBS existentes, 10 delas desenvolviam atividades do PCT através de equipes ESF e foram incluídas no estudo; excluiu-se a unidade que não desenvolvia açôes de controle da TB. Os gestores entrevistados e documentos oficiais, como os relatórios de gestáo disponibilizados, informavam que o PCT estava implantado nas 10 UBS supracitadas, mas não dispunham de um critério explícito para definir a condição de implantação. Para fins da coleta de dados aceitou-se o critério empírico localmente adotado: foram consideradas com PCT implantado as unidades que desenvolvam açóes de controle de TB, independentemente do tipo.

As entrevistas foram realizadas mediante roteiro estruturado versando sobre temas correlatos às dimensôes de avaliação adotadas na matriz de julgamento. Os roteiros constaram de 30 questóes sobre estrutura das unidades, perfil e qualificação dos recursos humanos, 25 questôes sobre operacionalização das açôes e vigilância epidemiológica, e 60 questôes sobre o processo de gestão. Além das supracitadas entrevistas, os itens que compóem a Matriz de Julgamento foram usados como um roteiro complementar de entrevista, tendo igualmente orientado a observaçáo, que acompanhou as rotinas de atendimento de TB na UBS, priorizando-se os aspectos correspondentes às dimensôes contidas na matriz de julgamento. Trata-se, portanto, de observaçáo focada no objeto (Minayo, 2004). e registrada em caderno de campo (Minayo, op.cit). As entrevistas foram gravadas e as anotaçóes oriundas das observaçôes foram categorizadas e ordenadas em estruturas de análise relevantes para os temas priorizados na pesquisa (Gomes et al., 2005). A análise dos dados qualitativos foi efetuada a partir de aproximaçóes sucessivas entre o material coletado e o objeto e objetivos do estudo, visando dissociar a totalidade em partes, de modo a facilitar a análise e apreender os sentidos atribuídos ao PCT, e à sua operacionalização, pelos profissionais de saúde. A essas informaçôes foram acrescentadas notas e interpretaçôes preliminares realizadas a partir da observação e registradas em caderno de campo (Gaskell, 2008). As entrevistas foram codificadas, de modo a garantir o anonimato dos entrevistados, sendo depois transcritas integralmente. Os dados das entrevistas seráo objeto de análise detalhada em outra publicação. Para fins do presente artigo seu conteúdo foi utilizado somente para elucidar alguns aspectos do funcionamento das açôes do PCT contribuindo para aprimorar o processo avaliativo e, particularmente, para apreender o entendimento dos sujeitos sobre esse contexto e as relaçôes sociais onde se insere o PCT em Coari e as potenciais incongruências entre a norma e a "práxis" dos profissionais envolvidos na ação programática em tela (Minayo, 2004).

Para a seleção de entrevistados, foram consideradas a categoria profissional e as funçôes desempenhadas no PCT, seja na gestão, seja na assistência. $\mathrm{O}$ município dispunha, na rede básica, de sete médicos, dez enfermeiros das Equipes de Saúde da Família, uma técnica de enfermagem, dois farmacêuticosbioquímicos e o gerente do programa, sendo todos incluídos no estudo; foram excluídos somente os profissionais que não atuavam no PCT.

Para o cálculo da população do município no período procedeu-se a interpolaçáo geométrica dos dados populacionais disponíveis no do Departamento de Informática do Sistema Único de Saúde (DATASUS) para 1991 e 2009, estratificados por faixa etária, sexo e procedência (urbana e rural), atendendo às recomendaçôes de Jannuzzi (2001). Para o cálculo das taxas de incidência utilizou-se a fórmula padrão ((casos/ população)*100.000), subtraindo-se as notificações excluídas, no período sob análise. Os dados foram organizados no programa Microsoft Excel 2003, e apresentados em tabelas por meio de frequência e percentual.

O desenho investigativo contou com modelo lógico (Figura 1) adaptado de Gattinara et al. (1995) que propiciou a apreensão da estrutura, processo e resultado da atenção programática no sistema municipal de saúde de Coari, com ênfase no Programa Saúde da Família (PSF). Deste modelo não foi utilizado o componente de eficácia, por falta de dados.

A avaliação do grau de implantaçáo do programa foi operacionalizada com base em Denis e Champagne (1997) por meio de matriz de julgamento adaptada de Oliveira e Natal (2007), contendo sete dimensóes de avaliação: contexto externo, contexto organizacional, estrutura (UBS), recursos humanos, vigilância epidemiológica, operacionalização das açôes do PCT e efetividade. Tais dimensôes foram pontuadas segundo critérios e indicadores de avaliação programática, podendo alcançar o máximo de 100 pontos, em caso de desempenho plenamente satisfatório. A atribuição de pontuação a cada dimensão avaliada foi um procedimento 


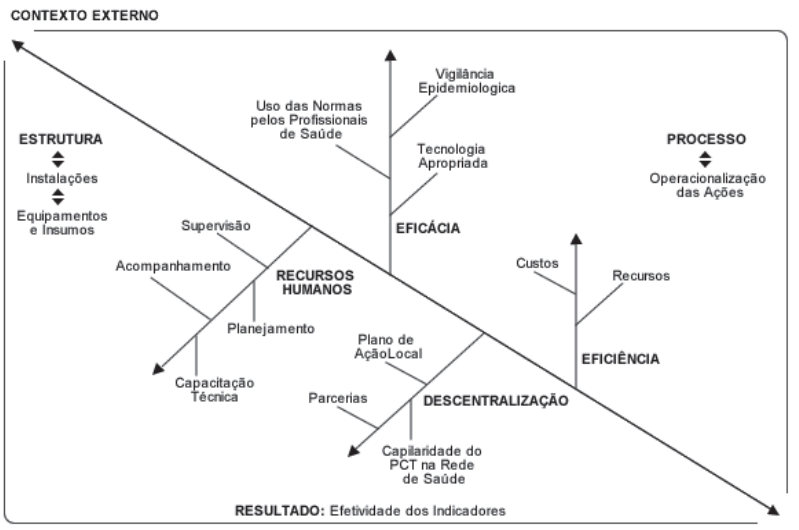

Figura 1 - Modelo lógico para avaliação do PCT de Coari.FONTE: Adaptado de Gattinara et al. (1995)

adaptado de Natal et. al (2004), pontuando-se cada categoria de análise de acordo com a sua relativa importância nas rotinas do programa. Assim, dimensōes como Vigilância Epidemiológica e Operacionalização das Açôes, que representam as atividades-fim do PCT receberam atribuição máxima de pontuaçấo de 20 e 25 pontos, respectivamente; outras dimensóes que representam atividades-meio ou elementos de contexto receberam pontuaçóes que variaram entre 10 e 15 . O procedimento gerou a seguinte classificação de grau de implantaçấo do PCT: 1 - incipiente $(\leq 33,3 \%), 2$ intermediário (> 33,3\% e $\leq 66,6 \%)$, e 3-avançado (> 66,6\%) (Vieira-da-Silva et al. 2007). As fontes usadas para a coleta de dados sobre cada dimensão da Matriz de Julgamento estão descritas na própria matriz (Tabela 2).

A pesquisa foi aprovada no Comitê de Ética/CEP-UFAM (CAAE N ${ }^{\circ}$ 0267.0.115.000-09).

\section{RESULTADOS E DISCUSSÃO}

A população urbana de Coari (64,6\% da população total do município) teve o percentual mais elevado de notificaçóes de TB $(74,1 \%)$, gerando, para o período, taxa média de incidência de 27 casos por 100.000 habitantes, contra 9,4 casos por 100.000 habitantes da área rural (Tabela 1).

No conjunto das notificaçôes os casos novos predominaram entre homens (com 104 em 170 casos ou 61,2\% das notificaçóes) gerando uma razão masculino/feminino de 1,6. A desproporção entre os sexos se manteve na comparaçáo de área urbana e rural, com taxas de incidência de 32,6/100.000 (homens urbanos) e 11,4/100.000 habitantes (homens de área rural). Já as mulheres tiveram 21,3/100.0000 e 7,4/100.0000, respectivamente (Tabela 1 ).

Quanto à idade, a maior incidência se deu em homens $(115,8 / 100.000$ habitantes) maiores de 60 anos de área urbana, seguido das faixas etárias de 40 a 59 anos $(60,2 / 100.000$ habitantes), de 20 a 39 anos (46,7/100.000 habitantes). Tais dados influenciam na incidência geral, mas acompanham a mesma ordem de incidência nas faixas etárias femininas. Ressalte-se ausência de casos notificados entre zero e nove anos, e apenas 36 casos em indivíduos de 10 a 19 anos (Tabela 1).

Do total de 170 casos novos, 95,5\% (164/170) foram de forma clínica pulmonar, havendo crescimento das notificaçôes entre 2001 a 2004 e oscilação da incidência registrada nos anos de 2005 a 2008.

A avaliação do contexto externo, a primeira dimensão avaliativa da matriz de julgamento, mostrou que em Coari o PCT é gerenciado pela Coordenaçáo do Programa Saúde da Família (PSF) que o considera implantado em 100\% das UBS (Tabela 2); o PCT tem pouca complementaridade com os outros setores ou programas, seja intra ou intersetorialmente. A gerência do programa não participa na definição dos recursos financeiros para o programa e desconhecia o teor da Portaria MS/3739/1998, que estabelece incentivo financeiro para alta por cura para tratamento supervisionado, baciloscopia diagnóstica e acompanhamento em TB (Tabela 2). Nesta dimensão também foi analisada a condição socioeconômica do usuário, verificando-se que, para o ano de 2000, 30,8\% da população do município náo tinha acesso a saneamento e

Tabela 1 - Distribuição dos casos novos e taxa de incidência por 100 mil habitantes de tuberculose por área de residência, faixa etária e sexo, Coari (AM), Brasil, 2001-2008.

\begin{tabular}{|c|c|c|c|c|c|c|c|c|c|c|c|c|c|c|c|}
\hline \multirow{3}{*}{$\begin{array}{l}\text { Faixa. } \\
\text { Etária } \\
\text { (anos) }\end{array}$} & \multicolumn{3}{|c|}{ População média ' } & \multicolumn{6}{|c|}{ Casos ii } & \multicolumn{4}{|c|}{ Taxa de incidência } & \multicolumn{2}{|c|}{ Taxa Geral } \\
\hline & \multirow[b]{2}{*}{$M$} & \multirow[b]{2}{*}{$\mathrm{F}$} & \multirow[b]{2}{*}{ Total } & \multicolumn{3}{|c|}{ Urbano } & \multicolumn{3}{|c|}{ Rural } & \multicolumn{2}{|c|}{ Urbano } & \multicolumn{2}{|c|}{ Rural } & \multirow{2}{*}{ Urbano } & \multirow{2}{*}{ Rura } \\
\hline & & & & $M$ & $\mathrm{~F}$ & total & $\mathrm{M}$ & $\mathrm{F}$ & total & $M$ & $\mathrm{~F}$ & $M$ & $\mathrm{~F}$ & & \\
\hline $0-4$ & 4200 & 4072 & 8272 & 0 & 0 & 0 & 0 & 0 & 0 & * & * & * & * & * & * \\
\hline $5-9$ & 4336 & 4172 & 8508 & 0 & 0 & 0 & 0 & 0 & 0 & * & * & * & * & * & * \\
\hline $10-19$ & 7136 & 7306 & 14442 & 14 & 12 & 26 & 5 & 5 & 10 & 24,5 & 20,5 & 8,7 & 8,5 & 22,5 & 8,6 \\
\hline $20-39$ & 8834 & 8851 & 17685 & 33 & 20 & 53 & 10 & 4 & 14 & 46,7 & 28,2 & 14,1 & 5,6 & 37,5 & 9,9 \\
\hline $40-59$ & 3736 & 3260 & 6996 & 18 & 8 & 26 & 8 & 5 & 13 & 60,2 & 30,7 & 26,8 & 19,2 & 46,4 & 23,2 \\
\hline 60 ou + & 1294 & 1107 & 2401 & 12 & 9 & 21 & 4 & 3 & 7 & 115,8 & 101,7 & 38,6 & 33,9 & 109,3 & 36,4 \\
\hline Total & 29536 & 28768 & 58304 & 77 & 49 & 126 & 27 & 17 & 44 & 32,6 & 21,3 & 11,4 & 7,4 & 27,0 & 9,4 \\
\hline
\end{tabular}

Fontes: i: DATASUS, 2001 a 2008 e IBGE, 2008. ii: SINAN, 2008. * Sem notificação. $M$ = Masculino; $F$ = Feminino. 
a taxa de analfabetos era de $31,5 \%$, entre maiores de 15 anos (IBGE 2000). Para esta dimensão o grau de implantação do programa foi considerado intermediário (Tabela 2).

$\mathrm{Na}$ avaliação da dimensão contexto organizacional os indicadores selecionados, mostraram que as açóes de controle da TB restringem-se à sede do município, sem programação, metas ou estratégias dirigidas à área rural. A implantação incipiente desta dimensão foi reforçada pelo não reconhecimento, em $50 \%$ das equipes de saúde da família, do Tratamento Diretamente Observado (TDO) como parte da rotina da unidade de saúde. Dentre os que reconheciam a importância do TDO, metade deles relatou não acompanhar nenhum paciente nessa modalidade de tratamento (Tabela 2).

Na dimensão estrutura física das UBS, detectou-se sala de vacina com BCG presente em $70 \%$ das unidades e estetoscópio e negatoscópio, em $60 \%$ destas. Condiçóes adequadas de ventilação e iluminação natural nos consultórios estavam presentes em apenas $17,5 \%$ das UBS observadas. As mesmas náo tinham laboratório para coleta e realizaçáo de baciloscopia de escarro, nem local específico para atendimento de sintomáticos respiratórios ou com diagnóstico de TB (Tabela 2). Como resultado desse item de avaliação encontrou-se grau de implantação incipiente.

$\mathrm{Na}$ dimensão recursos humanos o grau de implantação foi incipiente, dado que o planejamento das açóes é realizado periodicamente pela gerência, mas de forma não compartilhada com as equipes do PSF; o coordenador do PCT acumula as funçôes de gestão, deste e de outras açōes programáticas. A supervisão e a avaliaçấo de desempenho do programa não são feitas rotineiramente. Somente $33,3 \%$ dos profissionais de nível superior, e, $14,8 \%$ dos de nível médio, declararam ter sido capacitados para o controle de TB; tampouco no âmbito gerencial do programa houve referência à capacitação específica para a função (Tabela 2).

Na dimensão vigilância epidemiológica, houve referência à dificuldade em planejar, executar e monitorar as metas de controle e outros indicadores de vigilância epidemiológica da TB no município. Relatórios epidemiológicos são elaborados, mas não incluem indicadores operacionais do PCT e não são enviados a outros níveis do sistema de saúde. O SINAN está implantado somente no nível central, que conta com suporte de informática. Foi informado que a busca ativa de comunicantes é realizada rotineiramente, mas somente 30\% dos entrevistados responsáveis pela tarefa referiram realizar busca ativa faltosos, evidenciando uma contradição entre a norma técnica e a efetiva operacionalização delas na rotina do atendimento. Nesse item de avaliaçáo encontrou-se grau de implantação intermediário (Tabela 2).

$\mathrm{Na}$ operacionalização das ações do PCT os exames complementares disponíveis são o raio-X de tórax e a baciloscopia de escarro, cuja realização é centralizada no
Hospital Regional de Coari e no Laboratório Central de Análises Clínicas. Cultura e prova tuberculínica não são realizadas no município; a única referência disponível localiza-se em Manaus, a 363 km de distância. O teste HIV e a quimioprofilaxia estáo disponíveis para os usuários do PCT, em $70 \%$ e $50 \%$ das unidades, respectivamente; há disponibilidade de copos descartáveis e água para os pacientes em $85 \%$ das UBS. Os profissionais informam realizar atividades educativas em saúde, ainda que não disponham de material educativo específico para TB. Apesar da implantação do programa em $100 \%$ das unidades, $50 \%$ das equipes do PSF não investigam suspeitos de TB. A dimensão operacionalização das açôes foi avaliada como intermediária (Tabela 2).

A dimensão efetividade foi avaliada como incipiente, dado que o desfecho por cura foi 76,4\% (159) das notificações e o abandono foi 13,9\% (27) dos casos. (Tabela 2).

A avaliação de implantação do PCT do município de Coari, por meio das sete dimensóes selecionadas, cuja pontuaçáo totalizou 37,46 para o conjunto da matriz, permitiu identificar o grau de implantação do programa como intermediário, segundo os parâmetros estabelecidos pela pesquisa. Dado o número de notificaçóes de TB e informação sobre a descentralização do programa no município, se esperaria que ele estivesse mais bem estruturado para lidar com a TB. Pode-se inferir que a condição intermediária de implantaçáo também resulte em baixa capacidade do programa em captar os casos existentes, impedindo que o real problema da TB se expresse no perfil gerado a partir do SINAN.

Os casos de procedência urbana são predominantes. Em que pese a tendência de concentração dos casos de TB em aglomerados urbanos (Gonçalves et al. 2009; Hijjar et al. 2007), a centralizaçẫo das açôes do PCT na área urbana de Coari pode ter contribuído para ampliar essa diferença. O acesso do usuário da zona rural é dificultado pelas características amazônicas; em alguns casos, a pessoa precisa percorrer, viajando em pequenos barcos durante vários dias, cerca de $51 \mathrm{~km}$ para chegar ao atendimento na cidade (Vásquez 2008). Outra característica da população rural, que surpreende neste estudo, refere-se à concentração das notificaçóes, que comportam cerca de $1 / 3$ da taxa de incidência de TB em comparação à registrada na área urbana, com uma razão de taxas (urbano/rural) entre os sexos (masculino/feminino) de 1,6 associada a um quarto de abandono do tratamento para o sexo masculino da área rural.

$\mathrm{Na}$ distribuição da doença por faixa etária, as maiores taxas de incidência ocorrem a partir de 20 anos, e nas pessoas acima de 60 anos, revelando para os últimos, uma taxa com padrôes africanos (109,3/100mil habitantes) (WHO 2010). Tais achados são particularmente relevantes, por se tratar de um município no interior da floresta amazônica, com condições de vida diferentes dos grandes centros urbanos nos quais a 
circulação e aglomeraçáo contribuem para a elevação das taxas de TB (Gonçalves et al. 2009). Por outro lado, observou-se um "silêncio epidemiológico" em menores de 10 anos. Os entrevistados e documentos consultados não conseguem explicar as razóes para a ausência de notificaçóes nessa faixa etária, porém, ela pode estar ligada à falta de oferta de prova tuberculínica e cultura em Coari. A este limite se associa a grande distância de Manaus $(363 \mathrm{~km}$ em linha reta e $463 \mathrm{~km}$ por via fluvial, separam os dois municípios) - o local mais próximo para a oferta destes recursos diagnósticos. Tais exames são considerados os mais adequados para investigação de casos nessa faixa etária - o que dificulta a realização de diagnóstico de $\mathrm{TB}$ em crianças.

Diferente da situação do Brasil, com leve tendência de declínio na incidência de TB (Bierrenbach et al. 2007), o estado do Amazonas apresenta manutençáo dos níveis endêmicos entre 2004 e 2009 (Brasil 2009). Já em Coari não se pode fazer especulaçóes a respeito dos padróes de incidência da TB, tendo em vista as oscilaçóes encontradas no período do estudo. Tal disparidade de informaçóes é mais sugestiva de insuficiência na organizaçáo e oferta de serviços, como sugerem os resultados da avaliação, do que de mudanças no perfil epidemiológico. Embora o número de casos notificados tenha sido baixo, frente ao tamanho da população, ainda assim, os indicadores de desfecho de cura e abandono não alcançaram resultados satisfatórios, reforçando a idéia de pouca efetividade do PCT. Por outro lado, frente ao decréscimo da população no município nos quatro últimos anos poder-se-ia esperar que a incidência de TB, tomadas as notificaçóes como base, também decrescesse, o que não ocorreu.

A flutuação nas notificaçóes e nos valores das taxas anuais de incidência, associados ao não cumprimento das metas estipuladas pelo PNCT, demonstram dificuldades na operacionalização do programa, que reforçam a classificaçấo intermediária do grau de implantaçáo do PCT em Coari, conforme os achados deste estudo (Tabela 2).

A articulação entre o PCT e o PSF é assistemática e há baixa participaçấo das ESF no planejamento das ações do programa; não há fluxo de informação ou de avaliação, unilateral ou compartilhada, entre os programas. Tal como detectado em estudo anterior na Amazônia Legal (Natal et al. 2004), as parcerias com outros setores ou programas são incipientes e informais, dificultando um uso racional de recursos financeiros e humanos e reduzindo o rendimento do PCT.

Coari apresenta baixa proporção de população ocupada, além de elevada migração rural-urbana de pessoas com baixos índices de escolaridade. As instalaçôes sanitárias também têm baixo percentual de utilização nos domicílios, em comparação à média do estado e do país (Almeida e Souza 2008). Esse contexto gera um cenário favorável à disseminação da TB, tal como visto em avaliaçôes anteriores do PCT dirigidas a outros municípios (Natal et al. 2004; Moreira et al. 2006; Oliveira e Natal 2007).

No que diz respeito à estrutura física, os dados encontrados confirmam os achados de outros estudos (Moreira et al. 2006; Oliveira e Natal 2007; Natal et al. 2004), como estrutura inadequada das salas de atendimento, ausência de condiçóes para o diagnóstico de TB e comprometimento da biossegurança. Tais dificuldades de estrutura podem forçar, como parece ocorrer em Coari, a centralização dos serviços, pois profissionais e pacientes tendem a acorrer a locais com melhores condiçôes para diagnóstico e atendimento (Moreira et al., 2006). No município estudado, apesar do discurso explícito de descentralizaçâo, o que se constatou foi a centralização das açôes em uma única unidade de saúde, num movimento inverso às diretrizes do SUS e à política do controle da TB, que definem a atenção básica como principal vetor da operacionalizaçáo do PNCT. A indefiniçáo de critérios definidores da implantação do programa em unidades descentralizadas favorece a persistência da centralizaçáo e da baixa efetividade do PCT, prevalecendo a categoria implícita que faz equivaler descentralização à realização de umas poucas e fragmentárias açóes de controle da TB pelas ESF que atuam nas UBS.

A desarticulação e incipiente descentralizaçâo das açôes de TB encontradas na rede básica eram tributárias de problemas cotidianos dos serviços, tais como: a) dificuldade dos profissionais da rede básica, particularmente os médicos, de assumirem a doença como um agravo de responsabilidade da atenção básica. Os depoimentos deixaram evidente que, para os médicos, o diagnóstico e tratamento de TB era atribuição da unidade hospitalar e não da unidade básica de saúde; b) o pouco reconhecimento, pelos profissionais de saúde, da estratégia TDO; e c) a não disponibilidade de recursos diagnósticos e terapêuticos em todas as unidades de saúde.

As açóes de vigilância epidemiológica, cujo escopo de atuação era orientado para gerar dados para o SINAN e gerar relatórios periódicos para a SEMSA-Coari, não orientavam a tomada de decisão nos processos de gestão. A baixa capacitaçấo das equipes de saúde e a ausência de sistema de informação nas UBS produziram subutilização da vigilância epidemiológica como ferramenta de monitoramento de metas e indicadores do PCT em Coari, de modo similar aos achados de Natal et al. (2004;2008) para outros municípios na Amazônia e fora dela. Esses achados reforçam a ideia de Bezerra et al. (2009), de que as práticas de vigilância têm representado dimensões secundárias no processo de operacionalizaçấo do PNCT e, denotam a necessidade de esforços adicionais, por PCT local, para efetivar a vigilância epidemiológica e a estratégia TDO.

Entretanto, faz-se necessário assinalar as limitaçóes da pesquisa, marcada pela ausência de importantes instrumentos 
Tabela 2 - Matriz de Julgamento do Programa de Controle de Tuberculose de Coari, 2001-2008

\begin{tabular}{|c|c|c|c|c|c|c|}
\hline Dimensão & Critérios/Indicadores & Tipo de coleta & Pontos máximos & $\begin{array}{r}\Sigma \text { da po } \\
N\end{array}$ & $\begin{array}{l}0 \text { obtida } \\
\%\end{array}$ & $\begin{array}{c}\text { Grau de } \\
\text { Implantação }\end{array}$ \\
\hline \multirow{5}{*}{ 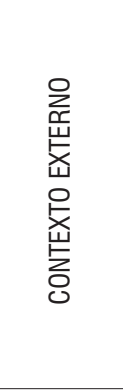 } & $\begin{array}{l}\text { Porcentagem de equipes de PSF com PCT } \\
\text { implantado. }\end{array}$ & $\begin{array}{c}\text { Dados } \\
\text { secundários } \\
\text { (SIAB) e entrevista }\end{array}$ & 4 & 04 & 100 & \multirow{5}{*}{ II } \\
\hline & $\begin{array}{l}\text { Recebe incentivo financeiro (bônus) de acordo } \\
\text { com a portaria } 3739 / 98 \text {. }\end{array}$ & Entrevista & 2 & 00 & 0 & \\
\hline & $\begin{array}{l}\text { Integração com outros programas e/ou } \\
\text { instituições. }\end{array}$ & Entrevista & 2 & 0,6 & 30 & \\
\hline & Condição sócio-econômica do usuário & $\begin{array}{l}\text { Dados } \\
\text { secundários }\end{array}$ & 2 & 00 & 0 & \\
\hline & subtotal & - & 10 & 4,6 & 46,0 & \\
\hline \multirow{4}{*}{ 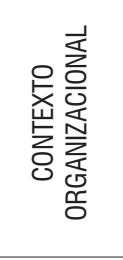 } & Conhece e utiliza a estratégia TD0. & Entrevista & 4 & 2 & 50 & \multirow{4}{*}{ I } \\
\hline & $\begin{array}{l}\text { A gerência do PCT participa na gestão do } \\
\text { recurso. }\end{array}$ & Entrevista & 3 & 0 & 0 & \\
\hline & $\begin{array}{l}\text { As ações do PCT são estendidas para } \\
\text { a área rural. }\end{array}$ & Entrevista & 3 & 0 & 0 & \\
\hline & subtotal & - & 10 & 2 & 20 & \\
\hline \multirow{6}{*}{ 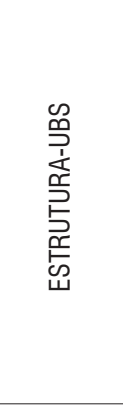 } & Local específico para atender o SR ou TB. & Entrevista e visita & 2 & 0 & 0 & \multirow{6}{*}{ I } \\
\hline & $\begin{array}{l}\text { Iluminação natural e ventilação nos } \\
\text { consultórios. }\end{array}$ & Entrevista e visita & 2 & 0,35 & 17,5 & \\
\hline & Sala de vacina. & Entrevista e visita & 2 & 1,4 & 70 & \\
\hline & $\begin{array}{l}\text { Equipamentos para exame de tuberculose: } \\
\text { 1. negatoscópio } \\
\text { 2. estetoscópio }\end{array}$ & Entrevista e visita & 2 & 1,2 & 60 & \\
\hline & $\begin{array}{l}\text { Laboratório para exame tuberculose, análise } \\
\text { clínica de BK. }\end{array}$ & Entrevista e visita & 2 & 0 & 0 & \\
\hline & subtotal & - & 10 & 2,95 & 29,5 & \\
\hline \multirow{10}{*}{ 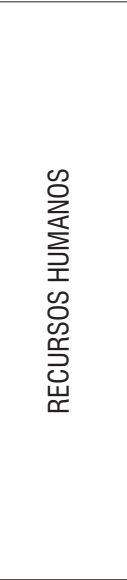 } & $\begin{array}{l}\text { Gerente (G) do PCT é exclusivo para o } \\
\text { programa }\end{array}$ & Entrevista & 1 & 0 & 0 & \multirow{10}{*}{ I } \\
\hline & G. capacitado em tuberculose. & Entrevista & 2 & 0 & 0 & \\
\hline & G. trabalha 40h no PCT. & Entrevista & 1 & 1,0 & 100 & \\
\hline & G. atua há mais de 03 anos no PCT. & Entrevista & 1 & 0 & 0 & \\
\hline & G. participa do planejamento municipal & Entrevista & 2 & 2,0 & 100 & \\
\hline & $\begin{array}{l}\text { G. faz supervisão/acompanhamento do } \\
\text { desempenho do PCT. }\end{array}$ & Entrevista & 2 & 0 & 0 & \\
\hline & $\begin{array}{l}\text { Equipe }(E) \text { de nível superior capacitada em } \\
\text { tuberculose. }\end{array}$ & Entrevista & 2 & 0,7 & 33,3 & \\
\hline & E. nível médio capacitada em tuberculose. & Entrevista & 2 & 0,3 & 14,8 & \\
\hline & $\begin{array}{l}\text { E. participa do planejamento e controle da } \\
\text { tuberculose. }\end{array}$ & Entrevista & 2 & 0,2 & 10 & \\
\hline & subtotal & - & 15 & 4,2 & 28 & \\
\hline \multirow{6}{*}{ 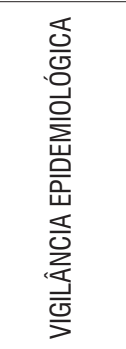 } & 0 SINAN está implantado no município. & Entrevista & 2 & 2,0 & 100 & \multirow{6}{*}{ II } \\
\hline & $\begin{array}{l}\text { Gerência (G) planeja anualmente os indicadores } \\
\text { e metas de controle da TB }\end{array}$ & Entrevista & 2 & 0 & 0 & \\
\hline & G. monitora os indicadores operacionais do PCT & Entrevista & 3 & 0 & 0 & \\
\hline & $\begin{array}{l}\text { G. avalia periodicamente o processamento e } \\
\text { análise dos dados }\end{array}$ & Entrevista & 2 & 2,0 & 100 & \\
\hline & $\begin{array}{l}\text { G. envia boletins epidemiológicos a outros } \\
\text { níveis }\end{array}$ & Entrevista & 2 & 0 & 0 & \\
\hline & G. emite relatórios & Entrevista & 1 & 1,0 & 100 & \\
\hline
\end{tabular}


Continuação - Tabela 2

\begin{tabular}{|c|c|c|c|c|c|c|}
\hline Dimensão & Critérios/Indicadores & Tipo de coleta & $\begin{array}{l}\text { Pontos } \\
\text { máximos }\end{array}$ & $\begin{array}{r}\Sigma \text { da po } \\
N\end{array}$ & $\begin{array}{l}\text { âo obtida } \\
\%\end{array}$ & $\begin{array}{l}\text { Grau de } \\
\text { Implantação }\end{array}$ \\
\hline \multirow{8}{*}{ 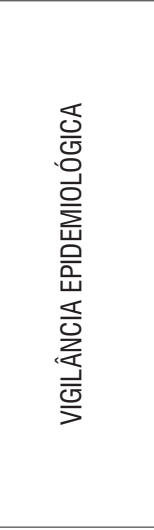 } & $\begin{array}{l}\text { G. dispõe de equipe de supervisão de } \\
\text { informática }\end{array}$ & Entrevista & 1 & 1,0 & 100 & \multirow{8}{*}{ II } \\
\hline & UBS notificam os casos de TB & Entrevista e visita & 1 & 0,1 & 10 & \\
\hline & Busca ativa de comunicantes & Entrevista & 1 & 0,9 & 90 & \\
\hline & Busca ativa de faltosos & Entrevista & 1 & 0,3 & 30 & \\
\hline & O SINAN está implantado nas UBS & Entrevista e visita & 1 & 0 & 0 & \\
\hline & O PCT local segue normas do PNCT & Entrevista e visita & 1 & 1,0 & 100 & \\
\hline & $\begin{array}{l}\text { E. realiza ações de vigilância: } \\
\text { 1. notificação }(0,4) \\
\text { 2. investigação }(0,4) \\
\text { 3. busca ativa }(0,4) \\
\text { 4. informação }(0,4) \\
\text { 5. imunização }(0,4)\end{array}$ & Entrevista e visita & 2 & 1,58 & 54 & \\
\hline & subtotal & - & 20 & 10,1 & 50,5 & \\
\hline \multirow{14}{*}{ 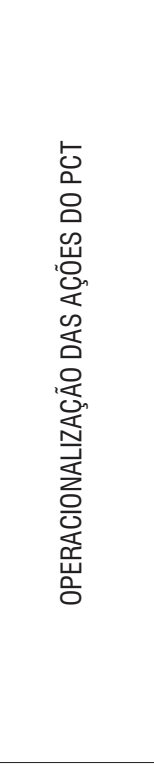 } & Disponibilidade na rede de esquema terapêutico & Entrevista e visita & 3 & 2,4 & 80 & \multirow{14}{*}{ II } \\
\hline & $\begin{array}{l}\text { Disponibilidade de água e copo descartável. } \\
\text { (DOTS) }\end{array}$ & Entrevista e visita & 1 & 0,85 & 85 & \\
\hline & Disponibilidade de quimioprofilaxia & Entrevista e visita & 2 & 1,0 & 50 & \\
\hline & Disponibilidade de baciloscopia & Entrevista e visita & 3 & 3,0 & 100 & \\
\hline & Disponibilidade de R-X & Entrevista e visita & 2 & 2,0 & 100 & \\
\hline & Disponibilidade de cultura & Entrevista e visita & 2 & 0 & 0 & \\
\hline & Disponibilidade de PPD & Entrevista e visita & 2 & 0 & 0 & \\
\hline & Disponibilidade de teste HIV & Entrevista e visita & 2 & 1,4 & 70 & \\
\hline & Disponibilidade mat. Educativo & Entrevista e visita & 2 & 0 & 0 & \\
\hline & Equipe $(\mathrm{E})$ realiza diagnóstico e tratamento & Entrevista & 2 & 1,4 & 71,4 & \\
\hline & E. acompanha TDO & Entrevista & 2 & 1,0 & 50 & \\
\hline & E. realiza educação em saúde: & & & & & \\
\hline & $\begin{array}{l}\text { 1. palestras }(0,4) \\
\text { 2. trab. com grupos }(0,4) \\
\text { 3.orientação individual }(0,4) \\
\text { 4. sala espera }(0,4) \\
\text { 5. no domicilio }(0,4)\end{array}$ & Entrevista & 2 & 0,56 & 28 & \\
\hline & subtotal & & 25 & 13,61 & 54,44 & \\
\hline \multirow{3}{*}{ 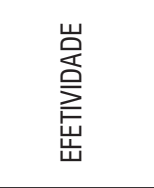 } & Cura & $\begin{array}{l}\text { Dados } \\
\text { secundários }\end{array}$ & 5 & 0 & 0 & \multirow{3}{*}{1} \\
\hline & Abandono & $\begin{array}{l}\text { Dados } \\
\text { secundários }\end{array}$ & 5 & 0 & 0 & \\
\hline & subtotal & & 10 & 0 & 0 & \\
\hline $\begin{array}{l}\text { Total de pontos } \\
\text { (todas as } \\
\text { dimensões) }\end{array}$ & & - & 100 & 37,46 & 37,46 & I \\
\hline
\end{tabular}

I - Incipiente; II - Intermediário

Fonte: Adaptado de Oliveira\& e Natal, 2008.

de gestáo local, como planos municipais de saúde e relatórios de gestâo para diversos anos e programaçóes locais orientassem as ações de controle da TB, o que restringiu o alcance das análises aqui empreendidas. Dentre estas, enfatizem-se os problemas na confiabilidade dos dados secundários disponibilizados, de resto, um problema comum a todas as análises que utilizam esse tipo de dado. Por outro lado, os limites na qualidade da informação podem ter contribuído para a obtenção de uma classificação em grau intermediário de implantação do programa neste município; assim, pode-se inferir que caso houvesse maior confiabilidade na capacidade 
de notificaçáo do sistema o cenário local poderia ainda ser pior do que o encontrado.

Por outro lado, os limites dos dados secundários foram contrabalançados pelos dados qualitativos primários, que serviram, dentre outras coisas, para levantar hipóteses sobre a qualidade da informação no município e evidenciar dimensões operacionais do PCT, que seriam inacessíveis mediante o uso exclusivo de dados secundários.

Por fim, assinale-se a frágil parceria das açôes do PCT com outras atividades das ESF, o que distancia as açôes programáticas do PCT do território em que o cuidado às famílias atendidas na rede básica é ofertado. A confluência das estratégias de gestão do PCT e PSF levaria à presunção de que as açóes de controle da TB estariam plenamente integradas no escopo de ação da atenção básica; porém, os resultados da pesquisa mostram o inverso.

\section{CONCLUSÕES}

A operacionalização do PCT é um desafio concreto para os serviços de saúde do município de Coari, seja por causas relacionadas ao cotidiano da populaçáo, como a precária rede saneamento básico, ou os baixos indicadores socioeconômicos que atuam como causas indiretas do aumento da $\mathrm{TB}$, seja pela inadequaçáo das ações de saúde ali ofertadas, as quais não atendem às necessidades de vigilância sistemática do PCT e às da população.

Os achados sugerem que o controle da TB é ação de baixa prioridade no sistema de saúde de Coari, apesar de um contexto externo adverso que favorece o aparecimento da doença e o abandono do tratamento, influenciando negativamente no sucesso do PCT. Tais fatores são agravados pela pouca utilização de atividades preventivas, pela fragmentação da atenção e pela centralizaçấo do diagnóstico da TB em uma única UBS, dificultando o acesso ao tratamento.

O contexto encontrado demanda a adoção de medidas amplas de reestruturaçâo no controle da TB em Coari, tais como a descentralização - de fato - das açóes do PCT, maior integração das ações programáticas na atenção básica, ampliação dos recursos diagnósticos e uso de inteligência epidemiológica para o monitoramento das açôes. Dentre as estratégias de melhoria também se fazem prioritárias, a capacitação de recursos humanos, a interiorização das açôes de saúde, particularmente dos programas específicos de controle de agravos de alta prevalência, o estímulo ao trabalho intersetorial e à reorganização da rede de saúde para um cuidado efetivo do portador de TB. Ainda que os dados obtidos - cujo caráter é prioritariamente local - não autorizem extrapolação das conclusóes para outras realidades, entende-se que os achados em Coari possam contribuir para uma reflexão sobre as dificuldades na operacionalização das açóes de controle da TB em outros municípios no Amazonas e no Brasil.

\section{BIBLIOGRAFIA CITADA}

Almeida, W.; Souza, N. 2008. Coari: Petróleo e Sustentabilidade - um exemplo amazônico. Desenvolvimento e Meio Ambiente. 17: 69-92.

Amazonas, 2008. Secretaria de Estado de Planejamento e Desenvolvimento Econômico. Perfil Municipal - Coari (AM). (http://www.seplan.am.gov.br/planejamento/ddr/ Condensadov3/Conteudo/subregiao7/12-coari.html). Acesso em 20/11/2008.

Barreira, D.; Grangeiro, A. 2007. Avaliação das estratégias de controle da tuberculose no Brasil. Revista de Saúde Publica. 41: 4-8.

Bezerra, L.C.A.; Freese, E.; Frias, P.G.; Samico, I.; Almeida, C.K.A. 2009. A vigilância epidemiológica no âmbito municipal: avaliação do grau de implantação das açôes. Cadernos de Saúde Pública. 25: 827-839.

Bierrenbach, A.L.; Gomes, A.B.F.; Noronha, E.F.; Souza, M.D.F.M. 2007. Incidência de tuberculose e taxa de cura, Brasil, 2000 a 2004. Revista de Saúde Publica. 41: 24-33.

Brasil, 2010a. Ministério da Saúde. Programa Nacional de Controle da Tuberculose. (http://www.opas.org.br/prevencao/site/UploadArq/ ProgramaTB.pdf). Acesso em 01/02/2010.

Brasil, 2010b. Ministério da Saúde. Secretaria de Atenção Básica. Sistema de Informação da Atenção Básica (SIAB). Indicadores da Atenção Básica. (http://siab.datasus.gov.br/SIAB/index. php?area=01). Acesso em 23/02/2010.

Brasil. 2009. Ministério da Saúde. Secretaria de Vigilância em Saúde. Sistema Nacional de Agravos de Notificação. Indicadores e dados Básicos. (http://tabnet.datasus.gov.br/cgi/deftohtm.exe?idb2009/ d0202.def). Acesso em 01/02/2010.

Contandriopoulos, A.P.; Champagne, F.; Denis, J.L.; Pineault, R. 1997. Avaliação na área da saúde: conceitos e métodos, p. 2931 In: Hartz, Z.M.A. (Ed.). Avaliação em saúde: dos modelos conceituais à prática na análise da implantação de programas. Rio de Janeiro: Fiocruz..

Cruz, A.K.; Teixeira, P. 2010. A dança dos números: a evolução demográfica dos municípios amazonenses a partir dos anos 70. Trabalho apresentado no XVII Encontro Nacional de Estudos Populacionais, realizado em Caxambu-MG - Brasil, de 20 a 24 de setembro de 2010.

Denis, J.L.; Champagne, F. 1997. Análise da implantação, p. 4988. In: Hartz, Z.M.A. (Ed.). Avaliação em saúde: dos modelos conceituais à prática na análise da implantação de programas. Rio de Janeiro: Fiocruz.

Gaskell, G. 2008. Entrevistas individuais e grupais, p. 64-89. In: Bauer, M.W.; Gaskell, G. (Ed). Pesquisa qualitativa com texto, imagem e som: um manual prático. Petrópolis: Vozes.

Gattinara, B.C.; Ibacache, J.; Puente, C.T.; Giaconi, J.; Caprara, A. 1995. Percepción de la comunidad acerca de la calidad de los servicios de salud públicos en los Distritos Norte e lchilo, Bolivia. Cadernos de Saúde Pública. 11: 425-438. 
Gomes, R.; Souza, E.R,; Minayo, M.C.S.; Malaquias, J.V.; Silva, C.F.R. 2005. Organização, processamento, análise e interpretação de dados: o desafio da triangulação, p. 185-221. In: Minayo, M.C.S.; Assis, S.G.; Souza, E.R. (Eds). Avaliação por triangulação de métodos: abordagem de programas sociais. Rio de Janeiro: Fiocruz.

Gonçalves, M.J.F.; Leon, A.C.P.; Penna, M.L.F. 2009. A multilevel analysis of tuberculosisassociated factors. Revista de Salud Publica. 11: 918-930.

Gonçalves, M.J.F.; Penna, M.L.F. 2007. Morbidade por tuberculose e desempenho do programa de controle em municípios brasileiros, 2001-2003. Revista de Saúde Publica. 41: 95-102.

Hijjar, M.A.; Gerhardt, G.; Teixeira, G.M.; Procópio, M.J. 2007. Retrospecto do controle da tuberculose no Brasil. Revista de Saúde Publica. 41: 50-57.

IBGE, 2000. Censo Demográfico 2000. Instituto Brasileiro de Geografia e Estatística. (www.ibge.gov.br). Acesso em $01 / 02 / 2010$.

IBGE, 2007. Contagem da População 2007. Tabelas de Resultados. População Recenseada e Estimada. (http://www.ibge.gov.br/ home/estatistica/populacao/contagem2007/defaulttab.shtm). Acesso em 22/12/2011.

Jannuzzi, P.M. 2001. Indicadores Sociais no Brasil: conceitos, fontes de dados e aplicaçôes para formulação e avaliação de políticas públicas. Campinas: Alínea. 146 pp.

Jamal, L.F.; Moherdaui F. 2007. Tuberculose e infecção pelo HIV no Brasil: magnitude do problema e estratégias para o controle. Revista de Saúde Publica. 41: 104-110.

Minayo, M.C.S. 2004. Fase do trabalho de campo, p. 105-196. In: Minayo, M.C.S. (Ed). O desafio do conhecimento: pesquisa qualitativa em saúde. São Paulo: HUCITEC.

Moreira, M.A.C.; Bello, A.S.; Melo, A.L.P.S.; Silva, M.V.; Lorusso, V. 2006. Análise de aspectos relacionados ao desempenho do Programa de Controle da Tuberculose em municípios do Estado de Goiás. Boletim de Pneumologia Sanitária. 14: 153-158.

Mourão, R.; Rivas, A.; Fraxe, T. 2007. O Estado da economia nas comunidades de várzea: atividades tradicionais e integraçáo de mercado, p. 149-170. In: Teixeira, P.; Brasil, M.; Rivas, A. (Eds). Produzir e viver na Amazônia Rural: estudo sociodemográfico de comunidades do Médio Solimôes. Manaus: EDUA.
Natal, S.; Penna, M.L.; Santos, E..L. 2004. Avaliação do Programa de Tuberculose: estudo de casos na Amazônia Legal. Boletim de Pneumologia Sanitária. 12: 91-109.

Natal, S.; Felisberto, E.; Alves, C.K.A. 2008. Aplicação do Método Rápido de Avaliação: proposta para auto-avaliação do Programa de Controle da Tuberculose. Revista Brasileira de Pneumologia Sanitária. 16: 74-84.

Oliveira, L.G.D.; Natal, S. 2007. Avaliação de implantação do Programa de Controle da Tuberculose no município de Niterói/ RJ. Revista Brasileira de Pneumologia Sanitária. 15: 29-38.

Souza, M.G.; Pinheiro, E.D.S. 2009. Incidência e distribuição da tuberculose na cidade de Manaus/AM, Brasil. Revista Geográfica Acadêmica. 3: 35-42.

Tanaka, O.Y.; Melo, C. 2000. Uma proposta de abordagem transdisciplinar para avaliação em Saúde. Interface-Comunicação, Saude, Educação. 4: 113-118.

Tanaka, O.Y.; Melo, C. 2004. Reflexões sobre a avaliação em serviços de saúde e a adoção das abordagens qualitativas e quantitativa, p. 121-136. In: Bosi, M.L.M. e Mercado, F.J. (Ed.). Pesquisa qualitativa de serviços de saúde. Petrópolis: Vozes.

Turato, G.R. 2003. As definições de amostra e de sujeito de pesquisa qualitativa, p. 351-368. In: Turato, G.R. (Ed.). Tratado de metodologia de pesquisa clínico-qualitativa. Petrópolis: Vozes.

Vásquez, F.G. 2008. Avaliação Epidemiológica da Hanseníase e dos Serviços Responsáveis por sua Assistência no Município de CoariAmazonas. Dissertação de Mestrado, Universidade Federal do Amazonas/Universidade Federal do Pará/Centro de Pesquisas Leônidas e Maria Deane-Fiocruz, Manaus, Amazonas. 107 pp.

Vieira-da-Silva, L.M.; Hartz, Z.M.A.; Chave, S.C.L.; Silva, G.A.P.; Paim, J.S. 2007. Análise da implantação da gestão descentralizada em saúde: estudo comparado de cinco casos na Bahia, Brasil. Cadernos de Saúde Pública. 23: 355-370.

WHO. 2010. World Health Organization. Global tuberculosis control: WHO report 2010. Geneva: World Health Organization.

Recebido em: 30-10-2011

Aceito em: 29-12-2011 